\title{
Severe intestinal dysbiosis is prevalent in primary Sjögren's syndrome and is associated with systemic disease activity
}

\author{
Thomas Mandl ${ }^{1,7^{*}}$ D, Jan Marsal ${ }^{2,3,4}$, Peter Olsson ${ }^{1}$, Bodil Ohlsson ${ }^{5}$ and Kristofer Andréasson ${ }^{6}$
}

\begin{abstract}
Background: Altered microbial composition of the intestine, commonly referred to as dysbiosis, has been associated with several autoimmune diseases including primary Sjögren's syndrome (pSS). The aims of the current study were to study the intestinal microbial balance in pSS and to identify clinical features associated with dysbiosis.
\end{abstract}

Methods: Forty-two consecutive pSS patients and 35 age-matched and sex-matched control subjects were included in the study in an open clinic setting. Stool samples were analyzed for intestinal dysbiosis using a validated 16S rRNAbased microbiota test (GA-map ${ }^{\mathrm{TM}}$ Dysbiosis Test; Genetic Analysis, Oslo, Norway). Dysbiosis and severe dysbiosis were defined in accordance with the manufacturer's instructions. Patients were evaluated with regard to disease activity (European League Against Rheumatism (EULAR) Sjögren's Syndrome Disease Activity Index (ESSDAI) and Clinical ESSDAI (ClinESSDAI)). In addition, patients were examined for laboratory and serological features of pSS as well as fecal calprotectin levels.

Furthermore, patients were investigated regarding patient-reported outcomes for pSS (EULAR Sjögren's Syndrome Patient Reported Index (ESSPRI)) and irritable bowel syndrome (IBS)-like symptoms according to the Rome III criteria.

Results: Severe dysbiosis was more prevalent in pSS patients in comparison to controls (21 vs 3\%; $p=0.018$ ). Subjects with pSS and severe dysbiosis had higher disease activity as evaluated by the ESSDAl total score (13 vs $5 ; p=0.049)$ and the ClinESSDAl total score (12 vs $5 ; p=0.049)$, lower levels of complement component 4 ( $0.11 \mathrm{vs} 0.17 \mathrm{~g} / \mathrm{L} ; p=0$. 004), as well as higher levels of fecal calprotectin (110 vs $33 \mu \mathrm{g} / \mathrm{g} ; p=0.001)$ compared to the other pSS patients. In contrast, severe dysbiosis among pSS patients was not associated with disease duration, IBS-like symptoms, or the ESSPRI total score.

Conclusions: Severe intestinal dysbiosis is a prevalent finding in pSS and is associated both with clinical and laboratory markers of systemic disease activity as well as gastrointestinal inflammation. Further studies are warranted to elucidate a potential causative link between dysbiosis and pSS.

Keywords: Primary Sjögren's syndrome, Microbiome, Gastrointestinal, Dysbiosis, Disease activity

\section{Background}

Primary Sjögren's syndrome (pSS) is a systemic autoimmune disease, characterized by lymphocytic infiltration of exocrine glands resulting in exocrine hypofunction and sicca symptoms. The disease may also result in various extraglandular manifestations (EGM)

\footnotetext{
* Correspondence: Thomas.mandl@med.lu.se

'Section of Rheumatology, Department of Clinical Sciences Malmö, Lund University, Malmö, Sweden

${ }^{7}$ Department of Rheumatology, Skane University Hospital Malmö, Inga Marie Nilssons gata 32, S-205 02 Malmö, Sweden

Full list of author information is available at the end of the article
}

such as arthritis, pulmonary involvement, renal disease, vasculitis, and neuropathy $[1,2]$. The gastrointestinal (GI) tract may also be involved and esophageal dysmotility [3, 4], gastroparesis [5], atrophic gastritis [6], and pancreatic insufficiency [6] are commonly encountered in pSS.

Increased levels of fecal calprotectin (F-calprotectin), a validated marker for GI inflammation, have been found in a subgroup of pSS patients and were associated with concomitant organic GI disease [7]. GI complaints are common in pSS and a large proportion of pSS patients 
exhibit symptoms typically observed in irritable bowel syndrome (IBS) or dysmotility $[5,8,9]$.

Recently, increasing interest has been directed against the importance of the GI microbiota and its influence on autoimmune disease [10]. In both health and disease, there is a continuous interaction between the microbiota, the epithelium, and the immune cells of the GI mucosa. This interaction has been suggested to have profound effects on both the local and systemic immune system [11], and may both curtail and amplify local and systemic inflammatory disease [12]. Because the GI epithelium is affected in pSS, through the inflammation of the exocrine glands as well as through diminished secretions, there are decreased levels of both protective and proliferative factors $[6,13,14]$. Consequently, the integrity of the epithelium and its barrier function may be compromised in pSS patients, possibly resulting in a perturbed microbiota-host immune system interaction.

An altered microbiota composition, commonly referred to as dysbiosis, has been shown to induce and modulate systemic inflammation in experimental animal models of rheumatic diseases [15], including pSS [16]. In the clinical setting, intestinal dysbiosis has been associated with several autoimmune diseases including rheumatoid arthritis, systemic lupus erythematosus, ankylosing spondylitis, celiac disease, autoimmune hepatitis, systemic sclerosis, and pSS [10, 15, 17-21]. Recently, de Paiva et al. demonstrated that intestinal dysbiosis may worsen experimental pSS in mice. They also reported an association between pSS and intestinal dysbiosis in a small number of patients $(n=10)$ [16].

The aims of this study were to explore intestinal microbial balance in pSS and to relate these findings to clinical features of disease.

\section{Methods \\ Patients}

Forty-two consecutive outpatients (median age 62, range 24-80 years; 40 females) diagnosed with pSS according to the American-European Consensus Group (AECG) criteria [22], but also fulfilling the American Congress of Rheumatology-European League Against Rheumatism (EULAR) criteria for pSS [23], seen at the Department of Rheumatology, Skane University Hospital, Malmö, Sweden, were included in the study. Concurrent inflammatory bowel disease (IBD) and antibiotic treatment during the last 3 months served as exclusion criteria. Patients' characteristics are presented in Table 1.

\section{Control group}

Thirty-five age-matched and sex-matched control subjects (median age 62, range 39-78 years; 33 females) consisting of hospital workers, their relatives, and their friends were invited and accepted for this study. Subjects
Table 1 Patient characteristics

\begin{tabular}{|c|c|}
\hline & pSS patients $(N=42)$ \\
\hline Age (years) & $62(51 ; 68)$ \\
\hline Sex (males/females) & $2 / 40$ \\
\hline Disease duration (years) & $16(7 ; 23)$ \\
\hline Current/prior/never smokers (\%) & 20/41/39 \\
\hline Anti-SS-A antibody seropositives (\%) & 76 \\
\hline Anti-SS-B antibody seropositives (\%) & 48 \\
\hline ANA seropositives (\%) & 79 \\
\hline RF seropositives (\%) & 52 \\
\hline $\lg G(g / L)$ & $13.1(10.0 ; 17.1)$ \\
\hline Complement component 3 (g/L) & $1.00(0.89 ; 1.15)$ \\
\hline Complement component 4 (g/L) & $0.16(0.12 ; 0.21)$ \\
\hline $\begin{array}{l}\text { Lip biopsy_focus score } \geq 1 \\
\text { (N positives/N studied (\%)) }\end{array}$ & 28/33 (85) \\
\hline ESSDAI total score & $6(1 ; 12)$ \\
\hline ESSPRI total score & $6(5 ; 8)$ \\
\hline Fecal calprotectin ( $\mu \mathrm{g} / \mathrm{g})$ & $38(20 ; 123)$ \\
\hline $\begin{array}{l}\text { Irritable bowel syndrome-like } \\
\text { symptoms (\%) }\end{array}$ & 42 \\
\hline Using proton pump inhibitors (\%) & 36 \\
\hline Using NSAIDs (\%) & 38 \\
\hline Using glucocorticoids (\%) & 36 \\
\hline$>5$ mg prednisolone daily & 7 \\
\hline 5 mg prednisolone daily & 24 \\
\hline$<5$ mg prednisolone daily & 5 \\
\hline Using anti-malarials (\%) & 29 \\
\hline Using other DMARDs (\%) & 2 \\
\hline
\end{tabular}

Demographic and clinical characteristics of the patients with primary Sjögren's syndrome ( $p S S)$. Results presented as median (interquartile range) or percentage of subjects

DMARD disease-modifying anti-rheumatic drug ESSDAl EULAR Sjögren's Syndrome Disease Activity Index, ESSPRI EULAR Sjögren's Syndrome Patient Reported Index, EULAR European League Against Rheumatism, NSAID nonsteroidal antiinflammatory drug, $A N A$ antinuclear antibodies, $R F$ rheumatoid factor

with any rheumatologic or IBD diagnosis, as well as concurrent antibiotic treatment, were excluded.

\section{Clinical assessment}

The patients were evaluated for systemic disease manifestations by the EULAR Sjögren's Syndrome Disease Activity Index (ESSDAI) [1] and the Clinical ESSDAI (ClinESSDAI) [24]. Patient-reported outcomes, including symptoms of sicca, pain, and fatigue, were evaluated by the EULAR Sjögren's Syndrome Patient Reported Index (ESSPRI) [25]. In addition, the presence of IBS-like symptoms, as defined by the Rome III criteria, was evaluated by a validated questionnaire on GI complaints [26]. Concomitant use of proton pump inhibitors (PPI), nonsteroidal anti-inflammatory drugs (NSAIDs), glucocorticoids 
(GCs), anti-malarials (AMA), and other disease-modifying anti-rheumatic drugs (DMARDs) was noted.

\section{Laboratory analyses}

Laboratory testing included measurement of levels of IgG, as well as analysis of autoantibodies including anti-SS-A and anti-SS-B antibodies, ANA, and RF. Complement levels were assessed by measurement of complement component 3 (C3) and complement component 4 (C4). F-calprotectin, a marker of GI inflammation, was measured with a commercially available enzyme-linked immunosorbent assay using a monoclonal antibody (Bühlmann Laboratories, Schönenbuch, Switzerland). All analyses were performed at the Department of Laboratory Medicine, Skane University Hospital.

\section{Evaluation of intestinal dysbiosis}

The GA-map ${ }^{\mathrm{Tm}}$ Dysbiosis Test (Genetic Analysis, Oslo, Norway) has been developed in order to identify and grade intestinal dysbiosis by analysis of microbial genes in a stool sample. The test makes use of 54 bacterial $16 \mathrm{~S}$ rRNA probes specific for various intestinal bacterial species or clades to generate data on the intestinal microbiota composition. Using a defined algorithm, these data are subsequently translated into a Dysbiosis Index Score (DIS) ranging from 1 to 5 . The test has been compared with MiSeq Illumina sequencing-based protocols and proven successful in identifying dysbiosis [21, 27-29]. In a previous study of a population consisting of young healthy adults, $84 \%$ exhibited DIS $1-2$ and $16 \%$ exhibited DIS $\geq 3$ [27]. In the current study, dysbiosis was defined as DIS $\geq 3$ and severe dysbiosis as DIS 5 , in accordance with the manufacturer's instructions.

\section{Ethics}

The study was approved by the Regional Ethics Review Board, Lund, Sweden (LU 2011/596). All patients and controls gave written informed consent and the study was performed in accordance with the declaration of Helsinki.

\section{Statistical analyses}

The Mann-Whitney $U$ test was used when comparing continuous variables and the chi-square test or Fisher's exact test for comparing discrete variables and frequency distribution analysis. Correlations were evaluated by Spearman rank correlation. Values are presented as median and interquartile range (IQR) or number and percentage of subjects. $p<0.05$ was considered statistically significant.

\section{Results}

Eubiosis and moderate dysbiosis were common in both the pSS group and the control group. However, the frequency distribution among the pSS patients was different from that of the controls $(p=0.045)$ (Fig. 1). A large subgroup of pSS patients exhibited severe dysbiosis (DIS 5) which was not the case among controls (21 vs $3 \%$; $p=0.018$ ) (Fig. 1). Consequently, we chose to analyze this pSS subgroup in relation to the other pSS subjects.

Patients with severe dysbiosis had higher disease activity, as evaluated by the ESSDAI, compared to the other pSS patients $(13(5 ; 16)$ vs $5(1 ; 10) ; p=0.049)$ (Fig. 2a). Similar findings were found for the ClinESSDAI $(12(4 ; 16)$ vs $5(0 ; 9) ; p=0.049)$. In line with these findings, hypocomplementemia was more pronounced amongst pSS patients with severe dysbiosis as reflected by lower serum levels of C4 $(0.11(0.07 ; 0.14)$ vs $0.17(0.14$; $0.21) \mathrm{g} / \mathrm{L} ; p=0.004$ ) (Fig. 2b). pSS patients with severe dysbiosis had significantly higher F-calprotectin (110 (61; 330) vs 33 (20; 74) $\mu \mathrm{g} / \mathrm{g} ; p=0.001$ ) (Fig. 2c) compared to the other patients. The associations remained when excluding patients with milder dysbiosis and comparing patients with DIS 5 only to patients with DIS 1-2 (data not shown).

Neither disease duration, smoking, classical symptoms of pSS (as evaluated by the ESSPRI), IgG, IgM, or IgA levels, or serological features of pSS was associated with dysbiosis or severe dysbiosis in pSS patients. IBS-like symptoms were not more common in pSS patients with severe dysbiosis (Table 2).

Usage of GCs was more common among patients with DIS 5 (67 vs 27\%; $p=0.049$ ). However, concomitant use of other medications (including the use of PPI, NSAIDs, and AMA) were not associated with severe or moderate dysbiosis in pSS patients (Table 2).

Subanalysis of the GA-map ${ }^{\text {тм }}$ Dysbiosis Test made it possible to explore the abundance of 15 separate clades or species of bacteria. A significantly higher fraction of pSS patients exhibited decreased levels of bacteria from the genera Bifidobacterium (38 vs 3\%; $p<0.001$ ) and Alistipes (19 vs 3\%; $p=0.017$ ) in comparison to control subjects. There was also a tendency toward lower levels of Faecalibacterium prausnitzii among the pSS subjects, but this difference was not statistically significant $(p=0.061)$.

\section{Discussion}

In the current study, we found that severe intestinal dysbiosis was a prevalent finding in pSS, affecting $21 \%$ of studied patients. In this cohort of consecutive pSS patients, severe intestinal dysbiosis was associated with both clinical and laboratory signs of systemic disease activity as well as with laboratory signs of GI tract inflammation, as evaluated by F-calprotectin.

Eubiosis and mild or moderate dysbiosis (DIS 1-4) were prevalent both in the pSS group and the control group. The relatively high fraction of intestinal dysbiosis 


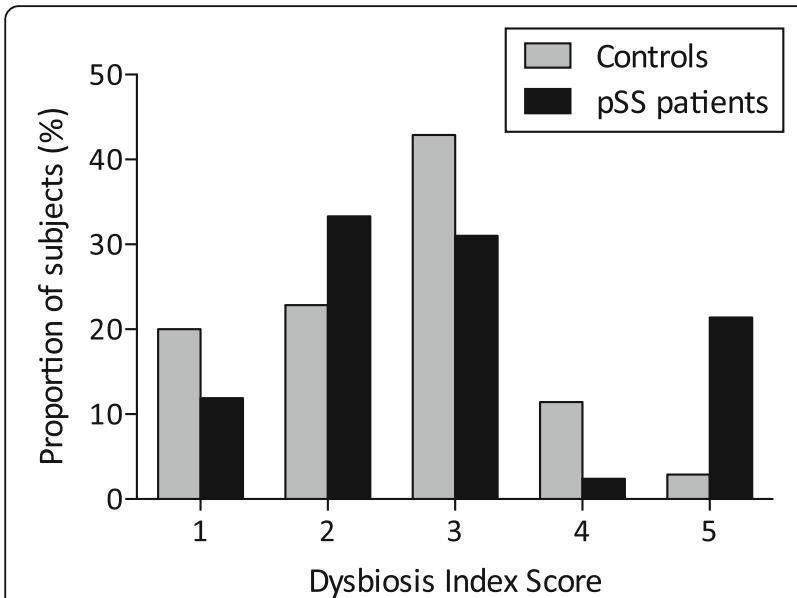

Fig. 1 Dysbiosis Index Score in primary Sjögren's syndrome and age-matched and sex-matched control subjects. A subgroup of patients with severe dysbiosis (DIS 5) can be identified among the subjects with primary Sjögren's syndrome (pSS) but not in the control group

in the control group differs from a previous report where only $16 \%$ of healthy adults exhibited DIS $\geq 3$ [27]. However, the controls described previously were younger (mean age 41 years) [27] than the age-matched and sex-matched controls of the current study (mean age 60 years). Also, in our control group we had few exclusion criteria (ongoing GI or rheumatic disease), which is in contrast to the previous report. However, severe intestinal dysbiosis was significantly more common in pSS patients $(21 \%)$ than in age-matched and sex-matched controls (3\%), indicating an altered intestinal microbial balance in a subgroup of pSS patients.

Intestinal dysbiosis has been found to be a feature of several rheumatic diseases, including rheumatoid arthritis, systemic lupus erythematosus, systemic sclerosis, and pSS $[10,16,20]$, as well as IBD [30]. Intestinal dysbiosis has hitherto been studied most extensively in IBD patients, in whom a decreased diversity in gut microbiota, increased numbers of bacteria driving inflammatory activity, as well as decreased numbers of bacteria with immunoregulatory effects have been demonstrated [30]. In rheumatic diseases as well as in IBD, it is still a matter of debate whether intestinal dysbiosis is a primary or secondary phenomenon of disease [31]. In animal models of IBD, loss of immunoregulatory bacteria and addition of disease-promoting bacteria contribute to disease activity, supporting a primary disease-driving role for intestinal dysbiosis in IBD [32]. Recently, two small studies reported oral [19] as well as intestinal [16] dysbiosis in pSS patients. In the prior study, the bacterial diversity was found to be lower in pSS patients, with normal salivary flow [19], thus implying that oral dysbiosis can occur irrespective of whether salivary flow was decreased or not. In the latter
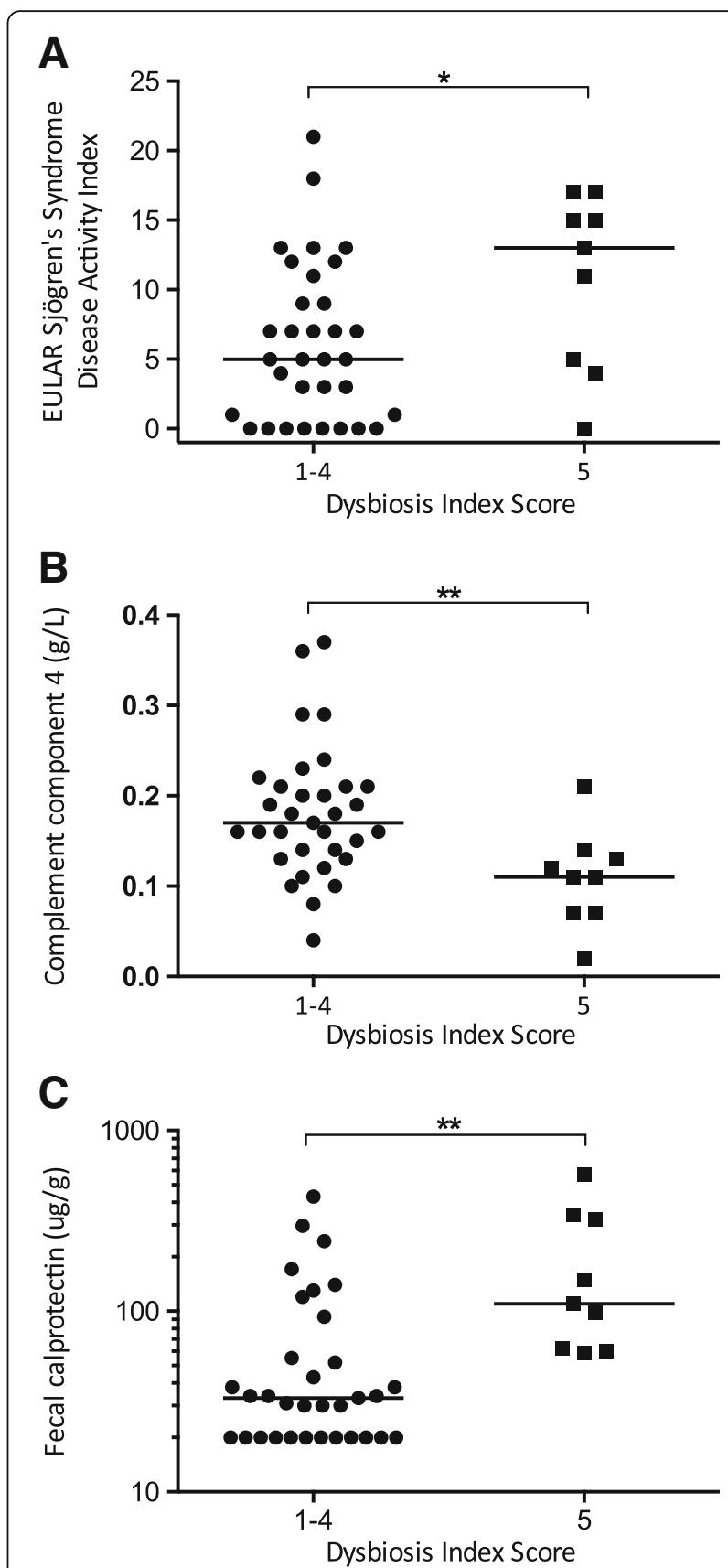

Fig. 2 Disease activity (a), hypocomplementemia (b), and intestinal inflammation (c) in pSS subjects with and without severe dysbiosis, defined as a Dysbiosis Index Score 5. Patients with intestinal

dysbiosis have higher ESSDAI scores (a), lower C4 levels (b), and higher levels of F-calprotectin (c) compared to other patients. Dot plot, median values marked by a horizontal line. ${ }^{*} p<0.05,{ }^{* *} p<0.005$. EULAR European League Against Rheumatism

study, signs of dysbiosis were found both in the oral cavity and in stool, and intestinal dysbiosis was associated with disease severity [16].

In the current study, we found that clinical disease activity, as evaluated by the ESSDAI and the ClinESSDAI, 
Table 2 Clinical features in patients with primary Sjögren's syndrome with and without severe dysbiosis

\begin{tabular}{llll}
\hline & Dysbiosis Index Score 1-4 (N=33) & Dysbiosis Index Score $5(N=9)$ & $p$ value \\
\hline Age (years) & $61(50 ; 68)$ & $62(58 ; 71)$ & 0.534 \\
ESSDAl total score & $5(1 ; 10)$ & $13(5 ; 16)$ & $0.049^{*}$ \\
ClinESSDAI total score & $5(0 ; 9)$ & $12(4 ; 16)$ & $0.049^{*}$ \\
ESSPRI total score & $6(5 ; 8)$ & $7(6 ; 8)$ & $12.4(10.2 ; 22.0)$ \\
IgG (g/L) & $13.2(10.0 ; 17.1)$ & $0.98(0.79 ; 1.10)$ & 0.224 \\
Complement component 3 (g/L) & $1.01(0.90 ; 1.21)$ & $0.11(0.07 ; 0.14)$ & 0.718 \\
Complement component 4 (g/L) & $0.17(0.14 ; 0.21)$ & 76 & 0.608 \\
Anti-SS-A antibody seropositives (\%) & 78 & 56 & $0.004^{* *}$ \\
Anti-SS-A antibody seropositives (\%) & 45 & 86 & 1.000 \\
Lip biopsy-focus score $\geq 1(\%)$ & 85 & 57 & $110(61 ; 330)$ \\
Irritable bowel syndrome-like symptoms (\%) & 39 & 67 & 0.714 \\
Fecal calprotectin ( $\mu$ g/g) & $33(20 ; 74)$ & 44 & 0.425 \\
Using glucocorticoids (\%) & 27 & 44 & $0.001^{* *}$ \\
Using anti-malarials (\%) & 24 & 33 & $0.049^{*}$ \\
Using proton pump inhibitors (\%) & 33 & 39 & 0.406 \\
Using NSAIDs (\%) & & 0.698 \\
\hline
\end{tabular}

Comparison of clinical characteristics in primary Sjögren's syndrome patients with severe dysbiosis (Dysbiosis Index Score 5) vs subjects with eubiosis or mild to moderate dysbiosis (Dysbiosis Index Score 1-4). Results presented as median (interquartile range) or fraction of patients with abnormal findings (\%)

ClinESSDAI Clinical EULAR Sjögren's Syndrome Disease Activity Index, ESSDAI EULAR Sjögren's Syndrome Disease Activity Index, ESSPRI EULAR Sjögren's Syndrome

Patient Reported Index, EULAR European League Against Rheumatism, NSAID nonsteroidal anti-inflammatory drug

${ }^{*} p<0.05$

${ }^{* *} p<0.01$

as well as laboratory signs of disease activity were associated with severe intestinal dysbiosis. These findings are reminiscent of the findings in the study by de Paiva et al. [16] showing an inverse correlation between fecal microbial diversity and combined ocular and systemic disease activity in pSS patients. In our study, patients with severe dysbiosis more often required treatment with lowdose GCs, which we suggest reflects systemic disease activity, but we cannot exclude that GC treatment causes dysbiosis. Indeed, some animal studies have indicated a direct effect of GCs on the microbial balance in the intestine [33, 34]. Of interest, treatment with PPI, NSAIDs, or AMA was not associated with severe intestinal dysbiosis in our study, reminiscent of findings in previous studies $[35,36]$.

F-calprotectin, a marker of GI inflammation, was significantly increased in pSS patients with severe intestinal dysbiosis, which is similar to findings in patients with systemic sclerosis [21], and suggests that an imbalance in microbiota in the gut of pSS patients coincides with GI inflammation. However, a potential causality remains to be determined and it can only be speculated whether intestinal dysbiosis drives the inflammatory process in pSS or is merely a consequence of systemic disease affecting the GI tract [37]. One hypothesis, however, is that an imbalance in the gut microbiota in the genetically susceptible individual leads to an increase in $\mathrm{T}_{\mathrm{H}} 17$ cells in the gut [38], which in turn enter the circulation and migrate to the exocrine glands, and possibly other tissues, to drive inflammation and development of both exocrine disease and EGMs in pSS.

We report low levels of bacteria from the genera Alistipes and Bifidobacterium in pSS patients. Interestingly, low levels of Alistipes have been reported previously in psoriatic arthritis and Crohn's disease [39, 40] and low levels of Bifidobacterium have been reported previously in both rheumatoid arthritis and Crohn's disease $[41,42]$. It remains to be explored why these specific alterations are found in clinically disparate diseases.

In contrast to patients with elevated F-calprotectin, patients with IBS-like symptoms did not display more severe dysbiosis than other patients. Instead, severe dysbiosis was as common among these subjects as in the control group. This finding is in contrast to previous reports in comparing IBS with healthy controls. The etiology of IBS and IBS-like symptoms is unknown, but is supposed to be multifactorial. Our findings may indicate that functional bowel symptoms associated with pSS have a different etiology compared to idiopathic IBS $[9,27]$.

To better understand the impact and importance of intestinal dysbiosis in pSS, future studies should investigate intestinal dysbiosis in larger samples of pSS patients. Ideally, we would like to study the intestinal microbiome longitudinally and in relation to factors likely to affect the intestinal microbiota (e.g., probiotics, 
prebiotics, antibiotics, specific diets, and anti-inflammatory medication). To further elucidate the important question of causality, further experimental animal studies in line with the previous publication by Paiva et al. [16] are warranted.

\section{Conclusions}

Severe intestinal dysbiosis is prevalent in pSS and associated with both clinical and laboratory markers of disease activity as well as with laboratory signs of GI inflammation.

\section{Abbreviations}

AECG: American-European Consensus Group; AMA: Anti-malarials;

C3: Complement component 3; C4: Complement component 4;

ClinESSDAl: Clinical EULAR Sjögren's Syndrome Disease Activity Index; DIS: Dysbiosis Index Score; DMARD: Disease-modifying anti-rheumatic drug; EGM: Extraglandular manifestations; ESSDAI: EULAR Sjögren's Syndrome Disease Activity Index; ESSPRI: EULAR Sjögren's Syndrome Patient Reported Index; EULAR: European League Against Rheumatism; F-calprotectin: Fecal calprotectin; GC: Glucocorticoid; IBD: Inflammatory bowel disease; IBS: Irritable bowel syndrome; NSAID: Nonsteroidal anti-inflammatory drug; PPI: Proton pump inhibitors; PSS: Primary Sjögren's syndrome

\section{Acknowledgements}

The authors would like to acknowledge the excellent assistance from study nurse Käth Nilsson as well as the Section of Clinical Immunology and Transfusion Medicine, Region Skåne, Lund, Sweden.

\section{Funding}

This study was supported by grants from the Swedish government to research in public health care (ALF), the Swedish Rheumatism Association, Region Skane, Anna-Greta Crafoord Foundation for Rheumatology Research, Lions Forskningsfond Skåne, Magnus Bergvalls Stiftelse, Swedish Society of Medicine, and Kock's Foundation.

\section{Availability of data and materials}

The datasets of the current study are available from the corresponding author on reasonable request.

\section{Authors' contributions}

TM and KA designed and organized the study, acquired and analyzed data, and prepared the manuscript. JM, PO, and $\mathrm{BO}$ contributed to analyzing data and reviewing the manuscript for intellectual content. All authors read and approved the final manuscript.

\section{Ethics approval and consent to participate}

The study was approved by the Regional Ethics Review Board, Lund, Sweden (LU 2011/596). All patients and controls gave written informed consent according to the declaration of Helsinki.

\section{Consent for publication}

Not applicable.

\section{Competing interests}

The authors of the study declare that they have no competing interests.

\section{Publisher's Note}

Springer Nature remains neutral with regard to jurisdictional claims in published maps and institutional affiliations.

\footnotetext{
Author details

${ }^{1}$ Section of Rheumatology, Department of Clinical Sciences Malmö, Lund University, Malmö, Sweden. ${ }^{2}$ Department of Gastroenterology, Skane University Hospital, Lund, Sweden. ${ }^{3}$ Immunology Section, Department of Experimental Medical Science, Lund University, Lund, Sweden. ${ }^{4}$ Section of Medicine, Department of Clinical Sciences Lund, Lund University, Lund, Sweden. ${ }^{5}$ Section of Internal Medicine, Department of Clinical Sciences Malmö, Lund University, Malmö, Sweden. 'Section of Rheumatology, Department of Clinical Sciences Lund, Lund University, Lund, Sweden.
}

${ }^{7}$ Department of Rheumatology, Skane University Hospital Malmö, Inga Marie Nilssons gata 32, S-205 02 Malmö, Sweden.

Received: 17 April 2017 Accepted: 3 October 2017

Published online: 24 October 2017

\section{References}

1. Seror R, Ravaud P, Bowman SJ, Baron G, Tzioufas A, Theander E, Gottenberg JE, Bootsma H, Mariette X, Vitali C. EULAR Sjogren's syndrome disease activity index: development of a consensus systemic disease activity index for primary Sjogren's syndrome. Ann Rheum Dis. 2010;69(6):1103-9. https:// doi.org/10.1136/ard.2009.110619.

2. Ramos-Casals M, Brito-Zeron P, Solans R, Camps MT, Casanovas A, Sopena B, Diaz-Lopez B, Rascon FJ, Qanneta R, Fraile G, Perez-Alvarez R, Callejas JL, Ripoll M, Pinilla B, Akasbi M, Fonseca E, Canora J, Nadal ME, de la Red G, Fernandez-Regal I, Jimenez-Heredia I, Bosch JA, Ayala MD, Morera-Morales L, Maure B, Mera A, Ramentol M, Retamozo S, Kostov B. Systemic involvement in primary Sjogren's syndrome evaluated by the EULAR-SS disease activity index: analysis of 921 Spanish patients (GEASSS Registry). Rheumatology (Oxford). 2014;53:321-31. https://doi.org/10. 1093/rheumatology/ket349.

3. Volter F, Fain O, Mathieu E, Thomas M. Esophageal function and Sjogren's syndrome. Dig Dis Sci. 2004;49(2):248-53.

4. Turk T, Pirildar T, Tunc E, Bor S, Doganavsargil E. Manometric assessment of esophageal motility in patients with primary Sjogren's syndrome. Rheumatol Int. 2005;25(4):246-9.

5. Hammar O, Ohlsson B, Wollmer $P$, Mand $T$. Impaired gastric emptying in primary Sjogren's syndrome. J Rheumatol. 2010;37(11):2313-8. https://doi. org/10.3899/jrheum.100280.

6. Sheikh SH, Shaw-Stiffel TA. The gastrointestinal manifestations of Sjogren's syndrome. Am J Gastroenterol. 1995;90(1):9-14.

7. Andreasson K, Ohlsson B, Mandl T. Elevated levels of faecal calprotectin in primary Sjogren's syndrome is common and associated with concomitant organic gastrointestinal disease. Arthritis Res Ther. 2016;18:9. https://doi.org/ 10.1186/s13075-015-0907-8.

8. Mandl T, Ekberg O, Wollmer P, Manthorpe R, Jacobsson LT. Dysphagia and dysmotility of the pharynx and oesophagus in patients with primary Sjogren's syndrome. Scand J Rheumatol. 2007;36(5):394-401. https://doi.org/ 10.1080/03009740701607638

9. Ohlsson B, Scheja A, Janciauskiene S, Mandl T. Functional bowel symptoms and $\mathrm{GnRH}$ antibodies: common findings in patients with primary Sjogren's syndrome but not in systemic sclerosis. Scand J Rheumatol. 2009;38(5):3913. https://doi.org/10.1080/03009740802709069.

10. van der Meulen TA, Harmsen $H$, Bootsma H, Spijkervet F, Kroese F, Vissink A. Oral Dis. 2016;22(8):719-734. doi:10.1111/odi.12472. Epub 2016 Apr 26. Review. PMID:26953630.

11. Honda K, Littman DR. The microbiota in adaptive immune homeostasis and disease. Nature. 2016;535(7610):75-84. https://doi.org/10.1038/nature18848.

12. Forbes JD, Van Domselaar G, Bernstein CN. The gut microbiota in immunemediated inflammatory diseases. Front Microbiol. 2016;7:1081. https://doi. org/10.3389/fmicb.2016.01081.

13. Koskenpato K, Ainola M, Przybyla B, Kouri VP, Virkki L, Koskenpato J, Ristimaki A, Konttinen $Y T$. Diminished salivary epidermal growth factor secretion: a link between Sjogren's syndrome and autoimmune gastritis? Scand J Rheumatol. 2016;45(2):118-21. https://doi.org/10.3109/03009742. 2015.1072243

14. Ebert EC. Gastrointestinal and hepatic manifestations of Sjogren syndrome. J Clin Gastroenterol. 2012;46(1):25-30. https://doi.org/10.1097/MCG. Ob013e3182329d9c

15. Scher JU, Littman DR, Abramson SB. Microbiome in inflammatory arthritis and human rheumatic diseases. Arthritis Rheumatol. 2016;68(1):35-45. https://doi.org/10.1002/art.39259.

16. de Paiva CS, Jones DB, Stern ME, Bian F, Moore QL, Corbiere S, Streckfus CF, Hutchinson DS, Ajami NJ, Petrosino JF, Pflugfelder SC. Altered mucosal microbiome diversity and disease severity in Sjogren syndrome. Sci Rep. 2016;6:23561. https://doi.org/10.1038/srep23561.

17. Hevia A, Milani C, Lopez P, Cuervo A, Arboleya S, Duranti S, Turroni F, Gonzalez S, Suarez A, Gueimonde M, Ventura M, Sanchez B, Margolles A Intestinal dysbiosis associated with systemic lupus erythematosus. MBio. 2014;5(5):e01548-14. https://doi.org/10.1128/mBio.01548-14. 
18. Costello ME, Ciccia F, Willner D, Warrington N, Robinson PC, Gardiner B, Marshall M, Kenna TJ, Triolo G, Brown MA. Intestinal dysbiosis in ankylosing spondylitis. Arthritis Rheumatol. 2015;67(3):686-691. doi:10.1002/art.38967.

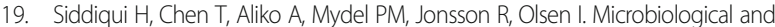
bioinformatics analysis of primary Sjogren's syndrome patients with normal salivation. J Oral Microbiol. 2016:8:31119. https://doi.org/10.3402/jom.v8.31119.

20. Szymula A, Rosenthal J, Szczerba BM, Bagavant H, Fu SM, Deshmukh US. T cell epitope mimicry between Sjogren's syndrome Antigen A (SSA)/Ro60 and oral, gut, skin and vaginal bacteria. Clin Immunol. 2014;152(1-2):1-9. https://doi.org/10.1016/j.clim.2014.02.004.

21. Andreasson K, Alrawi Z, Persson A, Jonsson G, Marsal J. Intestinal dysbiosis is common in systemic sclerosis and associated with gastrointestinal and extraintestinal features of disease. Arthritis Res Therapy. 2016;18(1):278. https://doi.org/10.1186/s13075-016-1182-z.

22. Vitali C, Bombardieri S, Jonsson R, Moutsopoulos HM, Alexander EL, Carsons SE, Daniels TE, Fox PC, Fox RI, Kassan SS, Pillemer SR, Talal N, Weisman MH. Classification criteria for Sjogren's syndrome: a revised version of the European criteria proposed by the American-European Consensus Group. Ann Rheum Dis. 2002;61(6):554-8.

23. Shiboski CH, Shiboski SC, Seror R, Criswell LA, Labetoulle M, Lietman TM, Rasmussen A, Scofield H, Vitali C, Bowman SJ, Mariette X, International Sjogren's Syndrome Criteria Working Group. 2016 American College of Rheumatology/European League Against Rheumatism Classification Criteria for Primary Sjögren's Syndrome: a consensus and data-driven methodology involving three international patient cohorts. Arthritis Rheumatol. 2017;69(1): 35-45. doi:10.1002/art.39859. Epub 2016 Oct 26. PMID: 27785888.

24. Seror R, Meiners P, Baron G, Bootsma H, Bowman SJ, Vitali C, Gottenberg JE, Theander E, Tzioufas A, De Vita S, Ramos-Casals M, Dorner T, Quartuccio L, Ravaud P, Mariette X, Force EST. Development of the ClinESSDAl: a clinical score without biological domain. A tool for biological studies. Ann Rheum Dis. 2016;75(11):1945-50. https://doi.org/10.1136/annrheumdis-2015-208504.

25. Seror R, Ravaud P, Mariette X, Bootsma H, Theander E, Hansen A, RamosCasals M, Dorner T, Bombardieri S, Hachulla E, Brun JG, Kruize AA, Praprotnik S, Tomsic M, Gottenberg JE, Devauchelle V, Devita S, Vollenweider C, Mandl T, Tzioufas A, Carsons S, Saraux A, Sutcliffe N, Vitali C, Bowman SJ. EULAR Sjogren's Syndrome Patient Reported Index (ESSPRI): development of a consensus patient index for primary Sjogren's syndrome. Ann Rheum Dis. 2011;70(6):968-72. https://doi.org/10.1136/ard.2010.143743.

26. Longstreth GF, Thompson WG, Chey WD, Houghton LA, Mearin F, Spiller RC. Functional bowel disorders. Gastroenterology. 2006;130(5):1480-91. https:// doi.org/10.1053/j.gastro.2005.11.061.

27. Casen C, Vebo HC, Sekelja M, Hegge FT, Karlsson MK, Ciemniejewska E, Dzankovic S, Froyland C, Nestestog R, Engstrand L, Munkholm P, Nielsen $\mathrm{OH}$, Rogler G, Simren M, Ohman L, Vatn MH, Rudi K. Deviations in human gut microbiota: a novel diagnostic test for determining dysbiosis in patients with IBS or IBD. Aliment Pharmacol Ther. 2015;42(1):71-83. doi:10.1111/apt. 13236. Epub 2015 May 14.

28. Magnusson MK, Strid H, Sapnara M, Lasson A, Bajor A, Ung KA, Ohman L. Anti-TNF therapy response in patients with ulcerative colitis is associated with colonic antimicrobial peptide expression and microbiota composition. J Crohns Colitis. 2016;10(8):943-52. https://doi.org/10.1093/ecco-jcc/jjw051.

29. Bennet SMP, Bohn L, Storsrud S, Liljebo T, Collin L, Lindfors P, Tornblom H, Ohman L, Simren M. Multivariate modelling of faecal bacterial profiles of patients with IBS predicts responsiveness to a diet low in FODMAPs. Gut. 2017. doi:10.1136/gutjnl-2016-313128. [Epub ahead of print].

30. Miyoshi J, Chang EB. The gut microbiota and inflammatory bowel diseases. Transl Res. 2017;179:38-48. doi:10.1016/j.trsl.2016.06.002. Epub 2016 Jun 14. Review. PMID:27371886.

31. Ni J, Wu GD, Albenberg L, Tomov VT Gut microbiota and IBD: causation or correlation? Nat Rev Gastroenterol Hepatol. 2017;14(10):573-584. doi:10. 1038/nrgastro.2017.88. Epub 2017 Jul 19.

32. Belkaid $Y$, Hand TW. Role of the microbiota in immunity and inflammation. Cell. 2014;157(1):121-41. https://doi.org/10.1016/j.cell.2014.03.011.

33. Stothart MR, Bobbie CB, Schulte-Hostedde Al, Boonstra R, Palme R, Mykytczuk NC, Newman AE. Stress and the microbiome: linking glucocorticoids to bacterial community dynamics in wild red squirrels. Biol Lett. 2016;12(1):20150875. https://doi.org/10.1098/rsbl.2015.0875.

34. Huang EY, Inoue T, Leone VA, Dalal S, Touw K, Wang Y, Musch MW, Theriault B, Higuchi K, Donovan S, Gilbert J, Chang EB. Using corticosteroids to reshape the gut microbiome: implications for inflammatory bowel diseases. Inflamm Bowel Dis. 2015;21(5):963-72. https://doi.org/10.1097/MIB. 0000000000000332.

35. Tsuda A, Suda W, Morita H, Takanashi K, Takagi A, Koga Y, Hattori M. Influence of proton-pump inhibitors on the luminal microbiota in the gastrointestinal tract. Clin Transl Gastroenterol. 2015;6:e89. https://doi. org/10.1038/ctg.2015.20.

36. Imhann F, Vich Vila A, Bonder MJ, Lopez Manosalva AG, Koonen DP, Fu J, Wijmenga C, Zhernakova A, Weersma RK. The influence of proton pump inhibitors and other commonly used medication on the gut microbiota. Gut Microbes. 2017:8(4):351-358. doi:10.1080/19490976.2017.1284732. Epub 2017 Jan 24.

37. Lopez P, Sanchez B, Margolles A, Suarez A. Intestinal dysbiosis in systemic lupus erythematosus: cause or consequence? Curr Opin Rheumatol. 2016; 28(5):515-22. https://doi.org/10.1097/BOR.0000000000000309.

38. Lopez P, de Paz B, Rodriguez-Carrio J, Hevia A, Sanchez B, Margolles A, Suarez $A$. Th17 responses and natural IgM antibodies are related to gut microbiota composition in systemic lupus erythematosus patients. Sci Rep. 2016;6:24072. https://doi.org/10.1038/srep24072.

39. Scher JU, Ubeda C, Artacho A, Attur M, Isaac S, Reddy SM, Marmon S, Neimann A, Brusca S, Patel T, Manasson J, Pamer EG, Littman DR, Abramson SB. Decreased bacterial diversity characterizes the altered gut microbiota in patients with psoriatic arthritis, resembling dysbiosis in inflammatory bowel disease. Arthritis Rheumatol. 2015;67(1):128-39. doi:10.1002/art.38892.

40. Willing BP, Dicksved J, Halfvarson J, Andersson AF, Lucio M, Zheng Z Jarnerot G, Tysk C, Jansson JK, Engstrand L. A pyrosequencing study in twins shows that gastrointestinal microbial profiles vary with inflammatory bowel disease phenotypes. Gastroenterology. 2010;139(6):1844-54. https:// doi.org/10.1053/j.gastro.2010.08.049. e1841.

41. Vaahtovuo J, Munukka E, Korkeamaki M, Luukkainen R, Toivanen P. Fecal microbiota in early rheumatoid arthritis. J Rheumatol. 2008:35(8):1500-5.

42. Favier C, Neut C, Mizon C, Cortot A, Colombel JF, Mizon J. Fecal beta-Dgalactosidase production and Bifidobacteria are decreased in Crohn's disease. Dig Dis Sci. 1997:42(4):817-22.

\section{Submit your next manuscript to BioMed Central and we will help you at every step:}

- We accept pre-submission inquiries

- Our selector tool helps you to find the most relevant journal

- We provide round the clock customer support

- Convenient online submission

- Thorough peer review

- Inclusion in PubMed and all major indexing services

- Maximum visibility for your research

Submit your manuscript at www.biomedcentral.com/submit
) Biomed Central 